\title{
A low fluctuation control strategy for PMSM direct drive system targeting Particle Beam Instrumentation Application
}

\author{
Jonathan Emery ${ }^{1}$, Patrik Andersson ${ }^{1}$, Federico Roncarolo ${ }^{1}$, Yann Thoma ${ }^{2}$
}

\begin{abstract}
Particle accelerators have a singular environment where multiple constraints are driving the engineering of equipment. Designers have to deal with the destructive effects of charged particles, high vacuum requirements, large temperatures and particular system architectures due to large-scale installations such as the Large Hadron Collider (LHC) at the CERN laboratory. At the same time, there is a continuous challenge to produce, measure and control smaller particle beams to increase the discovery potentials of large physics experiments. In this context, an innovative actuator has been built to measure precisely the size of beams down to $150 \mu m$ sigma, by moving a thin carbon wire of $30 \mu \mathrm{m}$ at about 20m $\cdot \mathrm{s}^{-1}$ through particle beams. Called Beam Wire Scanner (BWS), this system uses direct drive coupling to actuate a shaft inside a vacuum vessel without moving parts outside it. We are reporting on the design and validation of its control system based on torque control feedback as the only on-line closedloop system to operate this instrument. The proposed strategy keeps the smoothest action as possible on the system avoiding speed and position corrections that would lead to undesired torque variations, increasing the uncertainty of the carbon wire position.
\end{abstract}

\section{INTRODUCTION}

The Beam Wire Scanner (BWS) is an electromechanical system coupled to a particle detector to determine the transverse beam particles distribution inside accelerators. It uses a mechanism to move a thin carbon wire of $30 \mu \mathrm{m}$ located in vacuum at about $20 \mathrm{~m} \cdot \mathrm{s}^{-1}$ to go through the beam to produce a shower of secondary particles. A scintillator coupled to a photomultiplier tube evaluates this shower. The correlation between the carbon wire position and the number of secondary particles allows the determination of the beam size. Figure 1 shows the wire-scanner mechanism (left) hitting the proton beam with its carbon wire. The scintillator (center) detects the shower to evaluate the transverse beam particles distribution versus wire position, a distribution often looking like a typical Gaussian (right). The knowledge of the beam size is essential for accelerator operators and scientists to allow optimization of particle collisions at large physics experiments, such as the Compact Muon Solenoid (CMS) or A Toroidal LHC ApparatuS (ATLAS)

${ }^{1}$ J. Emery, Member, IEEE, F. Roncarolo, P. Andersson are with the European Organization for Nuclear Research (CERN), Beam instrumentation group, Geneva, Switzerland. contact: jonathan.emery@cern.ch

2 Y. Thoma is with the REDS Institute; HEIG-VD; HES-SO // University of Applied Sciences Western Switzerland, Switzerland. at CERN. The smaller the beams are, the higher is the probability of collisions leading to more potential discoveries.

\section{A. Wire-scanners operational controller}

There are three types of wire scanner mechanisms in operation across the CERN accelerators complex. From the relatively small machine called Proton Synchrotron Booster (PSB) [1] [2] to the very Large Hardron Collider (LHC), all these three different types are currently using Direct Current (DC) motors to generate the motion using the same controller. The position closed-loop uses a rotational or linear resistive sensor, depending on the target motion. The trajectory is pre-calculated off-line and is hardcoded in the controller as a look-up table. There is no dedicated control loop for the motor current. Figure 2 shows the closed-loop system. The trajectory lookup table in the controller $\theta_{\text {ref }}$ is compared to the measured position $\hat{\theta}(t)$. The error in position $e_{\theta}(t)=$ $\theta(t)-\widehat{\theta}(t)$ feeds a simple proportional controller $G_{c}(s)=$ $K_{c}$ which directly provides a speed request to the dc motor $G_{d} c(s)$ using a wide bandwidth linear operational amplifier considered perfect. The load is $G_{t}(s)$ and the position sensor is $K_{m \theta}$. This architecture does not allow the torque to be independently controlled. The speed reference directly comes from the position error, implying a constant error tracking. This architecture operates for more than a decade at CERN, providing sufficient performance for the existing mechanisms, but at the cost of reliability limitation [3].

\section{B. LHC Injectors Uprgade}

In the context of the Large Hadron Collider (LHC) Injectors Upgrade (LIU) project, a wire-scanner with

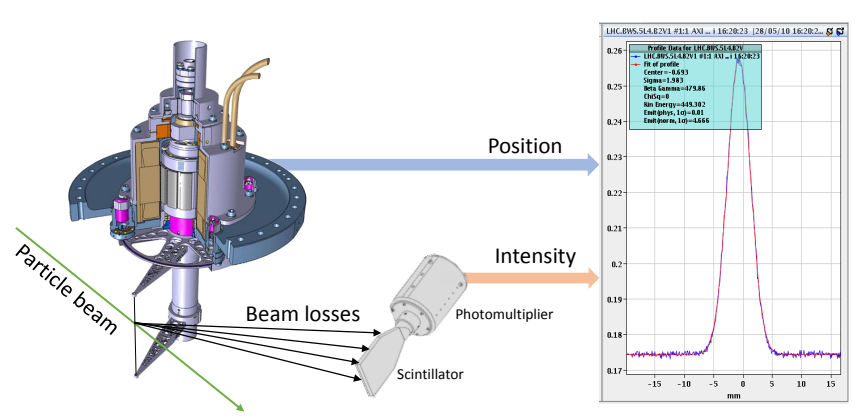

Fig. 1. Wire Scanner Principle with the electromechanical part (left), the particles detector (center) and the resulting signal (right) 


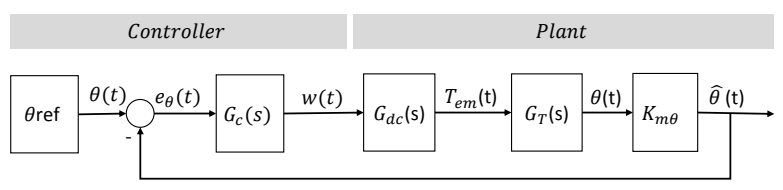

Fig. 2. Control architecture of the DC motor based wire-scanner

better performance than its predecessor is under design. The aim is to provide superior performance without the drawback of previous generation limitations. Old mechanisms are able to achieve precise measurements or high wire speed, but not both characteristics at the same time. Table I list the specification of the upgraded system. To meet these requirements, the innovative mechanism integrates a Permanent Magnet Synchronous Motor (PMSM) to have all moving parts inside the vacuum pipe, where particle beams travel, while stators are on the airside [4].

In 2015, the first prototype of this scanner was tested in the Super Proton Synchrotron (SPS) accelerator. To control this system, a controller based on field-oriented control (FOC) strategy is implemented on a custom electronics hardware [5]. This system provided good performance and reproducibility over time. The architecture of position-speed-current feedbacks relies on the shaft position for correct orientation of stator fields with respect to the shaft angle. During the operation of this system, a failure case of the controller has led to an incident that forced the accelerator to stop for 24 hours. Such an event has a high-cost impact to the whole accelerators complex. Since then different solutions are under investigation to improve system reliability, including the controller subsystem.

In 2018, for the prototype installed in the Proton Synchrotron (PS) accelerator, a second iteration of the electronics hardware operated the system with a different controller strategy. This strategy limits closed-loop feedbacks to the minimum necessary and relies mainly on an off-line calculation of feed-forward actions while keeping accurate torque control. An improvement in reliably is expected by reducing the complexity of the controller as well as by being able to provide a higher reproducibility of motions and smoother action to the mechanism.

\section{System Description}

The wire-scanner hardware has two geographically separated parts; the electromechanics and detectors are standing directly on the beamline of the accelerator, usually situated underground to avoid dangerous radiation to the environment. The control and power electronics are at the surface of accelerators to avoid damages to components induced by ionizing particles.

\section{A. Accelerator tunnel part}

The electromechanics is a direct drive system build around a Permanent Magnet Synchronous Motor (PMSM), a resolver for the absolute angular position, a magnetic braking system and an incremental high precision optical encoder. All these elements are located on the same side of the shaft. At the other end of the shaft, the measuring system is made of two forks and a stretched thin carbon wire in between. The in-house optical fiber-based incremental encoder achieves precise shaft angle measurement, used for the particle beam size calculation. The magnetic brake ensures that the system does not move without power. The connection of this scanner to its electronics is made through long copper cables up to $250 \mathrm{~m}$ to avoid the destructive effect of the charged particles to the electronics.

The motor is a frame-less three-phase PMSM wildly used for high dynamic drives as it develops a high ratio torque volume, ideal for this application. To apply the desired torque, the system needs the rotor angle given by the resolver to power correctly the three phases. To achieve high performance and simple controller design, this application uses a classical Field Oriented Control (FOC) strategy. This approach allows precise control by means of Clarke-Park transformation [6].

The motor model equations are:

$$
\begin{aligned}
\frac{d}{d t} i_{d} & =\frac{1}{L_{d}} u_{d}-\frac{R_{s}}{L_{d}} i_{d}+\frac{1}{L_{d}} \omega_{e}\left(L_{q} i_{q}\right) \\
\frac{d}{d t} i_{q} & =\frac{1}{L_{q}} u_{q}-\frac{R_{s}}{L_{d}} i_{q}-\frac{1}{L_{q}} \omega_{e}\left(L_{d} i_{d}+\Phi_{f}\right) \\
\frac{d}{d t} \omega_{m} & =\frac{\left(K_{T} i_{q}\right)-\omega_{m} B}{J} \\
\frac{d}{d t} \theta_{m} & =\omega_{m}
\end{aligned}
$$

being $u_{d}, u_{q}, i_{d}$ and $i_{q}$ the stator voltages and currents projected on the $d$ and $q$ axes, $R_{s}, L_{d}$ and $L_{q}$ the equivalent resistances and inductances, $\Phi_{f}$ the flux generated by the stator, $K_{T}$ the motor constant, $J$ the system inertia, $B$ its damping factor and $\omega$ and $\theta$, speed and angular position, respectively, expressed either in the electrical or in the mechanical frame.

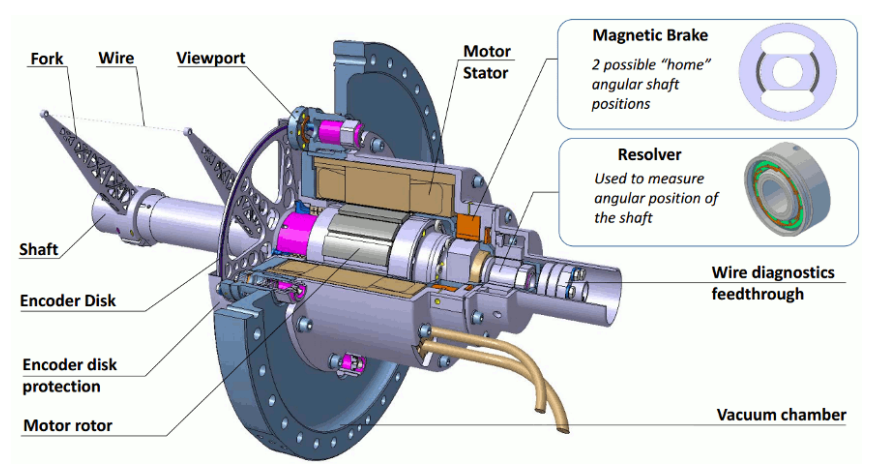

Fig. 3. Wire-scanner electromechanical details (courtesy of D. Gudkov) 
The magnetic braking system is a custom design developed for this application [7]. It uses reluctance forces to align the shaft to predefined safe positions for the thin carbon wire to be far away from the beam. It has one pair of permanent magnets on the stator and a ferromagnetic rotor with one pair of teeth, allowing two stable angular positions. This device behaves as a variable torque disturbance that is evaluated using a Finite Element Method (FEM) software to build a lookup table (LUT) of the torque disturbance versus shaft angle $T_{m b s}(\theta)$. Figure 4 presents the contribution of this system to the torque disturbance for each shaft angle. The maximum disturbance represents about $1 \mathrm{Nm}$ or $2 \%$ of the motor peak torque. Table I summaries the parameters used for the modelling of the system.
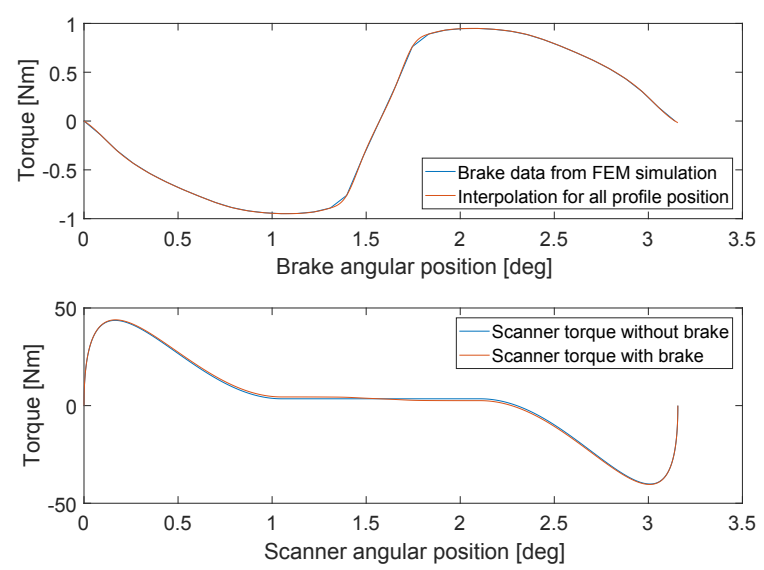

Fig. 4. Magnetic Breaking System torque in function of the shaft angle (top), and its effect on the total torque calculated to feedforward the system (bottom)

TABLE I

SYSTEM PARAMETERS FOR THE MODELLING

\begin{tabular}{c|c|c}
\hline Characteristics & Values & Units \\
\hline \hline C-Wire tangential speed $V_{n}$ & 20 & {$\left[\mathrm{~m} \cdot \mathrm{s}^{-1}\right]$} \\
Rotor nominal speed $W_{n}$ & 133 & {$\left[\mathrm{rad} \cdot \mathrm{s}^{-1}\right]$} \\
Position determination $W_{n}$ & 10 & {$[\mathrm{\mu m}]$} \\
Motor continuous torque $T_{c}$ & 14.6 & {$[N \cdot \mathrm{m}]$} \\
Motor peak torque $T_{p}$ & 55 & {$[N \cdot \mathrm{m}]$} \\
Motor Torque constant $K_{t}$ & 1.76 & {$\left[N \cdot \mathrm{m} \cdot A^{-1}\right]$} \\
Total inertia $J_{t}$ & $2.59 \cdot 10^{-3}$ & {$\left[\mathrm{~kg} \cdot \mathrm{m}^{2}\right]$} \\
Magnetic Breaking System & $T_{m b s}(\theta)$ & {$[N \cdot \mathrm{m}]$} \\
Static frictional force $T_{s f f}$ & 0.2 & {$[N \cdot \mathrm{m}]$} \\
Dynamic friction $D_{f}$ & 0.01 & {$\left[N \cdot \mathrm{m} \cdot \mathrm{rad} \mathrm{d}^{-1} \mathrm{~s}^{-1}\right]$} \\
Cable length $L_{c}$ & 185 & {$[\mathrm{~m}]$} \\
DC bus voltage $V_{d c}$ & 340 & {$[\mathrm{~V}]$} \\
Control loop freq. $F_{l o o p}$ & 16.129 & {$[\mathrm{kHz}]$} \\
Resolver accuracy & 1 & {$[\mathrm{deg}]$} \\
Optical encoder accuracy & 8 & {$[\mathrm{~mm}]$} \\
\hline
\end{tabular}

\section{B. Accelerator surface part}

The digital controller collects the angle of the shaft with a resolver as well as with an in house optical angular sensor based on optical fiber [8], it then calculates the new currents for the motor three phases and activates the power parts using pulse-width modulation (PWM). Many state variables are recorded at the rate of $16 \mathrm{kHz}$ in sync with the control loop calculation. The controllers are running inside a Field Programmable Gate Array (FPGA). This type of component is very flexible and allows unlimited parallel tasks in real-time, while microprocessors have to share time between real-time tasks.

\section{CONTROL DESIGN}

The architecture for the controller uses Field-Oriented Control (FOC), also called vector control. It is a variablefrequency drive (VFD) method where the stator currents of a three-phase AC electric motor are identified as two orthogonal components vector aligned to the rotor angle using Clark-Park transforms. In this reference frame, there are two components: the direct $d$-axis and the quadrature $q$-axis current. The direct component produces flux-weakening going against the permanent magnets and the quadrature component produces the torque. A coupling exist between the direct and the quadrature current which needs to be reduced to apply precise and smooth force to the system. This decoupling helps the controller to avoid high transients with wild spectral components that can excite the natural frequencies of the structure including the carbon wire [9].

\section{A. Current decoupling}

Since we are interested at low torque fluctuations, it is possible to decouple the interaction between the $d$-axis and $q-$ axis currents. The motor equations (1a) (1b) written in Laplace are (2a) (2b), the last contribution of each equation is canceled by using feedforward. For this purpose, new inputs in Eq. (2c) and (2d) are created.

$$
\begin{aligned}
s I_{d} & =\frac{1}{L_{d}} U_{d}-\frac{R_{s}}{L_{d}} I_{d}+\frac{1}{L_{d}} \omega\left(L_{q} I_{q}\right) \\
s I_{q} & =\frac{1}{L_{q}} U_{q}-\frac{R_{s}}{L_{q}} I_{q}+\frac{1}{L_{q}} \omega\left(L_{q} I_{q}+\phi_{f}\right) \\
U_{d}^{\prime} & =U_{d}-\omega_{e}\left(L_{q} I_{q}\right) \\
U_{q}^{\prime} & =U_{q}-\omega_{e}\left(L_{d} I_{d}+\phi_{f}\right)
\end{aligned}
$$

After the introduction of the decoupling bloc, the current loops simplify to the following convenient firstorder form:

$$
\begin{aligned}
& I_{d}=\frac{1}{L_{d} s}+R_{s} U_{d} \\
& I_{q}=\frac{1}{L_{q} s}+R_{s} U_{q}
\end{aligned}
$$

\section{B. Reference controller}

The first implementation of the controller uses a torque-speed-position closed-loop topology to control each variable individually. This implementation was successfully tested in the accelerator environment of the 
Super Proton Synchrotron (SPS), giving promising results [5] [6]. Figure 5 shows the controller architecture. There are three feedback loops; 1) motor currents feedbacks, 2) speed feedback, 3) position feedback. Because the application is primarily targeting trajectory tracking, and to minimize the steady-state tracking error, the controller includes feed-forwards pre-calculated for a given trajectory. To decouple the $d q$ components, a feed-forward uses the system speed to feed corrections to the other components. The speed is calculated by a Steady-State Kalman Filter (SSKF) from the position to produce better quality speed than a simple derivation [6].

\section{Optimized controller}

The main conceptual difference between earlier work on this wire-scanner and the controller presented in this paper is the absence of speed and position feedback, keeping only the currents feedback and decoupling feedforwards in charge. The goal is to simplify the controller implementation to increase reliability and lowering motion instabilities while ensuring smooth actuation of the scanner. The online actions of the controller, i.e. the closed-loop by feedbacks, are limited to the minimum necessary, avoiding correction of the position and speed. These simplifications are possible since the carbon wire does not need to be accurately positioned along the trajectory, but only accurately measured by a very fast high precision sensor. For the speed, there are minimum and maximum boundaries to respect for the correct operation of the system. Inside this window, the speed can vary smoothly without consequences.

Figure 6 presents the control architecture implemented for the Proton Synchrotron (PS) wire-scanner prototype. This controller is simpler compared to the previous implementation, focussing on the minimum necessary. Additionally to the position and speed feedback, the SSKF has been removed and the speed is directly taken from the Resolver to Digital Converter (RDC) internal processing, providing sufficient performances for the feed-forward decoupling of the $d$ and $q$ currents.

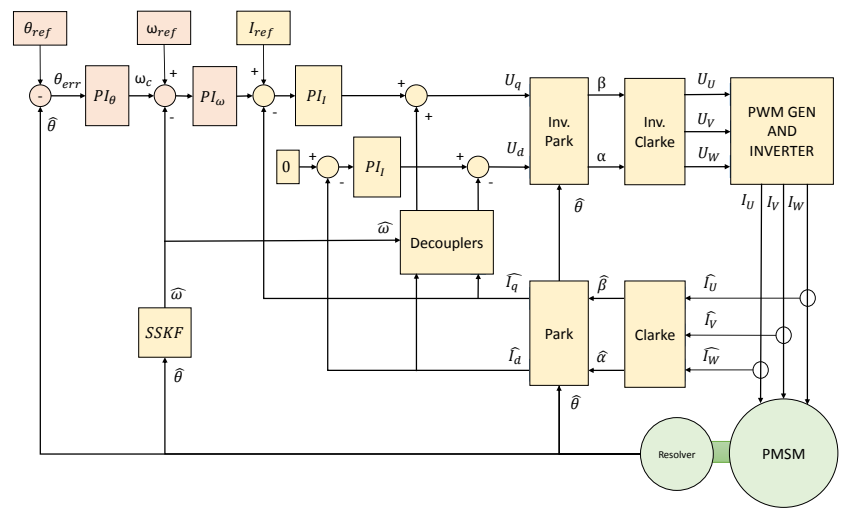

Fig. 5. Original control architecture used in the PSB and SPS accelerators

\section{Limitations}

This control strategy is not without drawbacks. Suppressing the position and speed feedbacks reveals potential modeling inaccuracies and parameters variation between the systems to control. As this controller will equip multiple replicas of the instrument, physical parameters variation may affect the performance. Like any physical system, each replica of the mechanism is having slightly different behavior due to the variation of components characteristics or to the way they are linked together:

- System inertia

- Static and dynamic frictions

- Motor torque constant incertitude

- Alignment motor-resolver

- Finite accuracy of the resolver

The electrical drive is not part of this list since the $d q$ currents are feedback to impose the expected torque, even in case of large electrical setup variations such as power cable length or DC bus value.

Without an appropriate strategy to mitigate the consequences of those parameters variations, the trajectory of the mechanism will be different for each replica, by its length and top speed.

The basic approach to mitigate this incertitude is to tune parameters for each replica. This approach was performed on two different replicas: one in our laboratory and the ones inside the PS accelerator. Without adaptation, the acceleration and deceleration do not have the same strength, as the system frictions are significantly different. During this trial and error session, it became also clear that a second table was needed to account for behavior differences between motion direction, i.e. the IN beam motion does not need the same strength than the OUT of the beam motion.

\section{E. off-line trajectory generation}

The particular constraints of operating this system with a powerful beam motivate the nominal trajectory to be of $\pi$ rad to avoid hitting the particle beam with the wire more than once per motion direction.

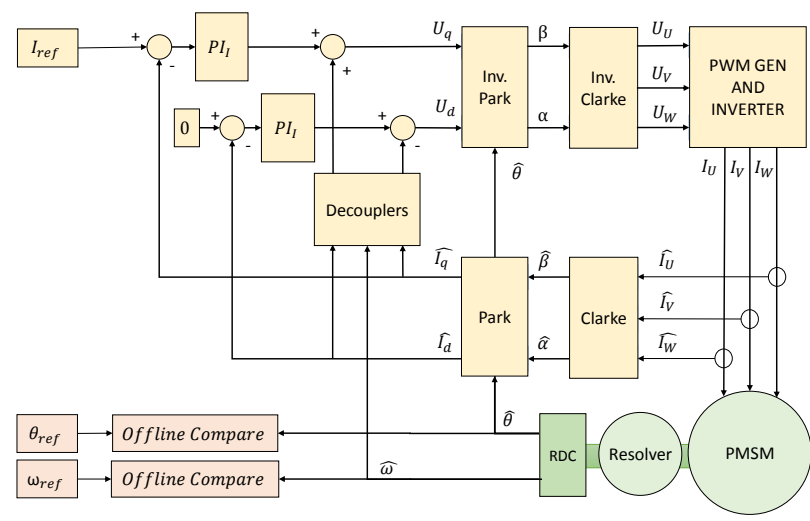

Fig. 6. Proposed control architecture for the PS accelerator 


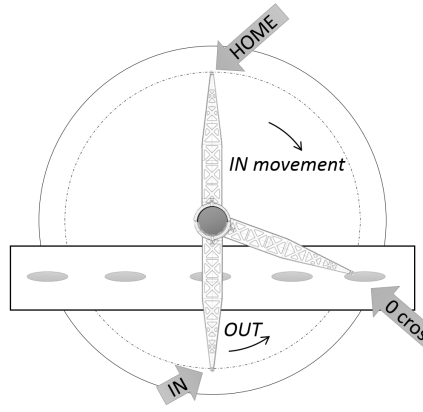

A.

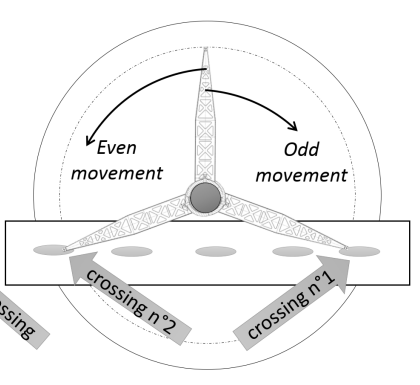

B.
Fig. 7. Nominal trajectory of the wire-mechanism (left) and an optimised one under consideration (right)

Figure 7 presents the forks angular positions during a measurement cycle as presented (left), while an alternative configuration is under consideration (right). The 'HOME' position is the initial angle, and represents the zero coordinate. The 'IN' position is the arriving angle after the first motion. After a defined waiting time, the system goes back to its initial 'HOME' angle.

The trajectory is constructed from the 3rd derivative of the position, known as the Jerk, to ensure smoothest forces applied to the mechanism [9]. It contains 3 phases: $P_{a}$ - one sinusoid period, $P_{b}$ - followed by a certain time without Jerk, $P_{c}$ - then a sinusoid period shifted by $\pi$ rad compared to $P_{a}$.

An iterative algorithm was developed to generate trajectories. It takes for inputs the predefined angles of the acceleration $\theta_{a}$ and deceleration $\theta_{b}$ and the target speed during the constant speed $P_{b}$, leaving the length of the constant speed as a free parameter. knowing the system inertia, frictions, and potential perturbations, it is possible to approximate the torque to be applied by the motor. the quadratic current in the rotor frame $q$ is determined by using the nominal motor torque constant Kt. The parameters used with the Algorithm 1 to generate the trajectory of the PS prototype are:

$\omega_{\text {target }}=70\left[\mathrm{rad} \cdot \mathrm{s}^{-1}\right], \theta_{\text {target }_{\text {acc }}}=\theta_{\text {target }_{\text {dec }}}=\frac{\pi}{3}[\mathrm{rad}]$, $J_{e r k_{\text {min }}}=4 e 5\left[\mathrm{rad} \cdot \mathrm{s}^{-3}\right]$, Jerk $k_{\text {step }}=1 e 3\left[\mathrm{rad} \cdot \mathrm{s}^{-3}\right]$, $J e r k_{\text {max }}=1 e 7\left[\mathrm{rad} \cdot \mathrm{s}^{-3}\right]$.

The calculation of the current $q$-axis look-up table is then:

$$
\begin{aligned}
& T_{\text {brake }}(t)=T_{m b s}(\theta(t)) \\
& T(t)=\alpha(t) \cdot J_{\text {tot }}+\omega(t) \cdot D_{f}+T_{\text {brake }}(t) \\
& I_{q}(t)=\frac{T(t)}{K_{t}}
\end{aligned}
$$

Figure 8 presents the jerk curve generated with the algorithm (top). This curve is the reference to calculate the acceleration (second from top), the speed (3rd from top) and finally the position (bottom).

\section{EXPERIMENTAL VALIDATION}

The controller was implemented inside an in-house control platform built around a field-programmable gate
Algorithm 1: Trajectory generation of the acceleration phase $P a$

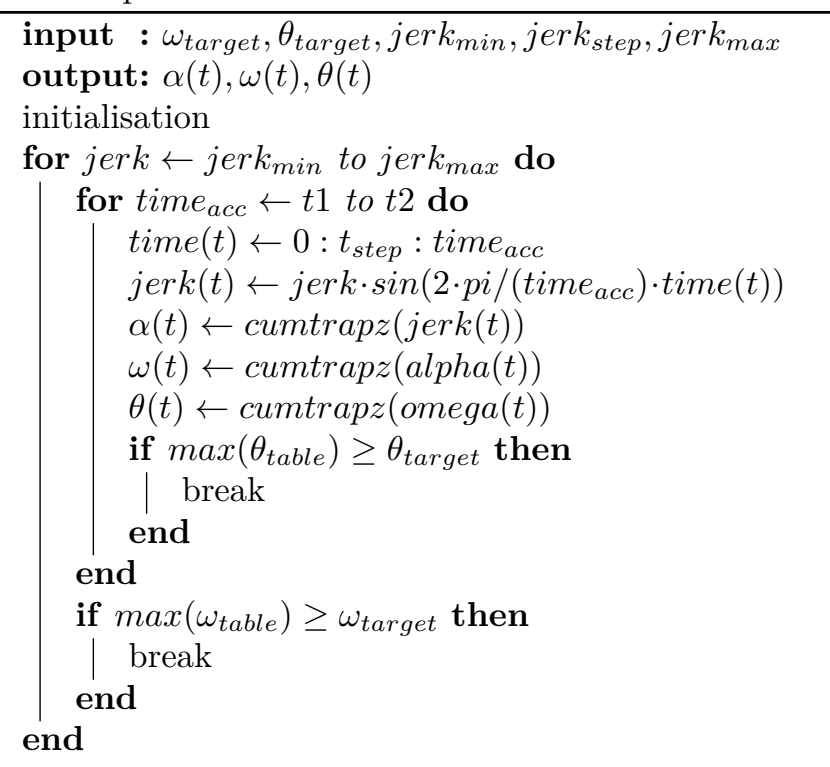

array (FPGA). This system was tested in the laboratory and in the Proton Synchrotron (PS) accelerator. This accelerator is a concrete environment where the system operates with a distance of about $190 \mathrm{~m}$ between the mechanism and the control, one of the longest distance foreseen. Figure 9 shows the accelerator position with the wire-scanner installed inside its vacuum vessel. Many nearby cables make the global electromagnetic environment noisy for low signals measured over long cables, such as the resolver in this application.

\section{A. Performance evaluation method}

The experimental results of the proposed controller in the PS accelerator are compared to field tests data of the reference controller coming from the Proton Synchrotron

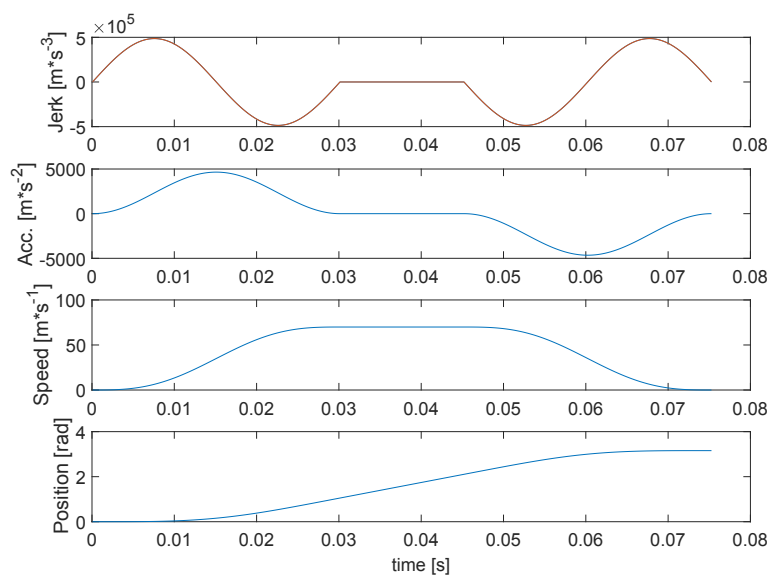

Fig. 8. Motion tables generated by the algorithm with the jerk (top), the acceleration (second), the speed (third) and the position (bottom) 


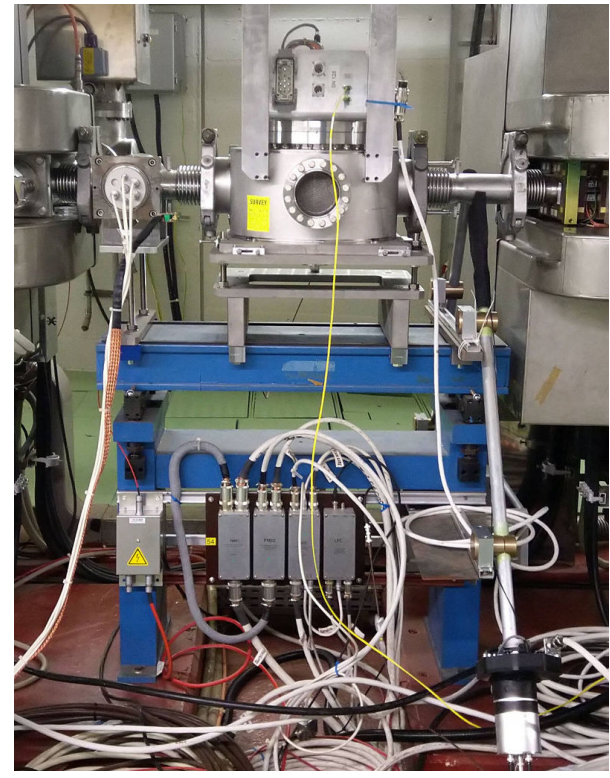

Fig. 9. Beam Wire Scanner prototype installed in the Proton Synchrotron (PS)

Booster (PSB) installation. The mechanics of the scanners are identical for the two setups.

The performances of the controllers are evaluated using their capability to minimise shot-by-shot variation for multiples events, the repeatability, and their ability to minimize the fluctuation of the driving force, here the rotor frame $q$-axis current. For each data set, the mean trajectory represents the reference signal. For each event, the difference is calculated and the Standard Deviation (SD) and the Absolute Maximum (AM) is extracted.

\section{B. Results}

There were more than three thousand measurement cycles performed with this controller in 2018. A subset of two hundred cycles was processed to compare it with the reference controller. The motion speed was limited at about $63 \%$ of the nominal speed to protect the prototype hardware. Later on, tests near the nominal speed were possible in the laboratory and are presented.

Figure 10 shows the motion cycles on top of each other obtained by the presented controller. The position (top) and the speed (middle) is given by the resolver while the current $q$-axis (bottom) is from the currents sensors processed by the controller.

The performance of each dataset is evaluated using the first half of the motion, from the OUT position at 0 [rad] to the IN position at around 3.14 [rad]. Figure 11 presents the data processing of the proposed controller current in the $q$-axis at the speed of $110\left[\mathrm{rad} \mathrm{s}^{-1}\right]$ (top) and the computed difference of each repetition with respect to the mean of all events (bottom).

Table II summarizes the tracking error, defined as the deviation from the mean, for each set of data generated by the optimized controller $(O p t)$ and the reference one $(R e f)$. There is a clear difference between these controllers. While the reference controller behaves better to track the angular position, the optimized controller presents a much lower shot by shot variation of the $q$ - axis current, meaning better torque repeatability applied to the mechanical system.

TABLE II

CONTROLlERS TRACKING ERROR

\begin{tabular}{c|c|c|c|c}
\hline Controller & events & variable & SD & AM \\
\hline \hline Ref @ 133 & 200 & & 0.042 & 0.1339 \\
Ref @ 133 & 9 & Position & 0.0278 & 0.068 \\
Opt @ 110 & 9 & [rad $]$ & $\mathbf{0 . 0 0 9 6}$ & $\mathbf{0 . 0 2 7 4}$ \\
Opt @ 70 & 193 & & 0.0804 & 0.2029 \\
\hline \hline Ref @ 133 & 200 & & 2.3658 & 9.7744 \\
Ref @ 133 & 9 & Speed & 1.8106 & 7.8157 \\
Opt @ 110 & 9 & {$\left[\mathrm{rad} \cdot \mathrm{s}^{-1}\right]$} & $\mathbf{0 . 4 3 9 4}$ & $\mathbf{2 . 3 2 2}$ \\
Opt @ 70 & 193 & & 2.0428 & 6.9218 \\
\hline \hline Ref @ 133 & 200 & & 3.9872 & 16.215 \\
Ref @ 133 & 9 & current $q-$ axis & 3.2691 & 16.5961 \\
Opt @ 110 & 9 & {$[\mathrm{~A}]$} & $\mathbf{0 . 1 1 4 9}$ & $\mathbf{0 . 7 3 3 1}$ \\
Opt @ 70 & 193 & & 0.2364 & 1.023 \\
\hline
\end{tabular}

\section{Time of flight verification}

The starting angle of the system varies from shot to shot when using the proposed controller without position feedback. This angle determines the wire arrival time on the particle beam. This timing is critical to measure precise moments of the acceleration process that can last no more than one millisecond. Figure 12 correlates the motion duration to hit the beam versus the starting angle (left). For the return motion, the timing is correlated with the maximum angle reached by the system. These data fit well linearly, making it simple to predict the motion duration using the starting angle. Another strategy

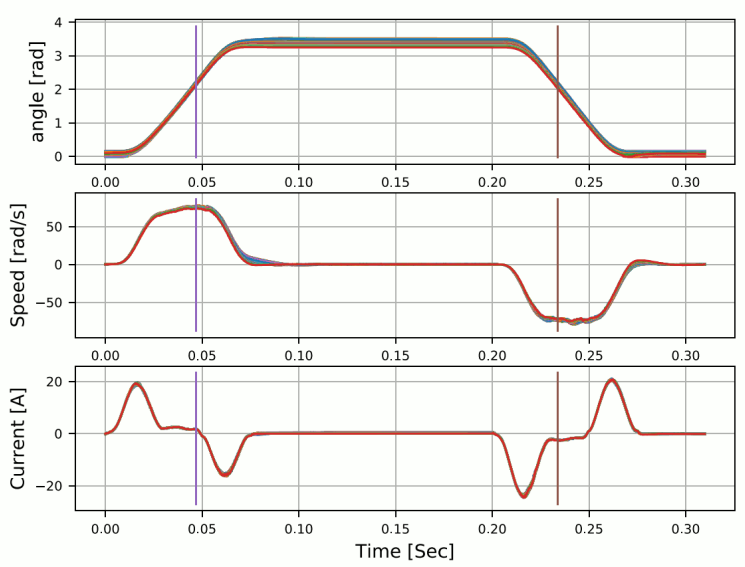

Fig. 10. Proposed controller tested in the Proton Synchrotron (PS) between the 2 and 15 October 2018. Shaft position (top), shaft speed (middle) and the rotating reference frame stator current $q$ for 194 cycles plot on top of each other 


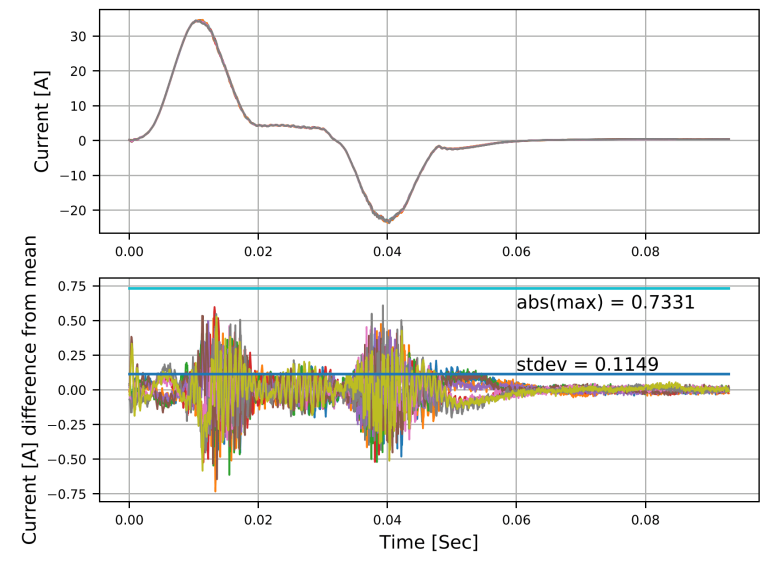

Fig. 11. Proposed controller tested in the laboratory at a nominal rotation of $110\left[\mathrm{rad} \cdot \mathrm{s}^{-1}\right]$ for 9 events with the current $q$ in the rotor frame (top) representing an approximation of the system torque, and the deviation of each event from the population mean value (bottom)

was tested to improve this timing. After each successful motion, one of the three motor coils is shortly powered to realign the rotor without position feedback and prepare for the next motion.

\section{CONCLUSION}

The beam wire scanner is an electromechanical system operating in particle beam accelerators to measure the beam size by means of a thin carbon wire actuated by a direct drive Permanent Magnet Synchronous Motor (PMSM) system. For this system, a controller based on Field Oriented Control (FOC) architecture was tested inside the Proton Synchrotron (PS) accelerator at CERN.

Unless its predecessor tested in other accelerators, this controller does not use any position or speed feedback.

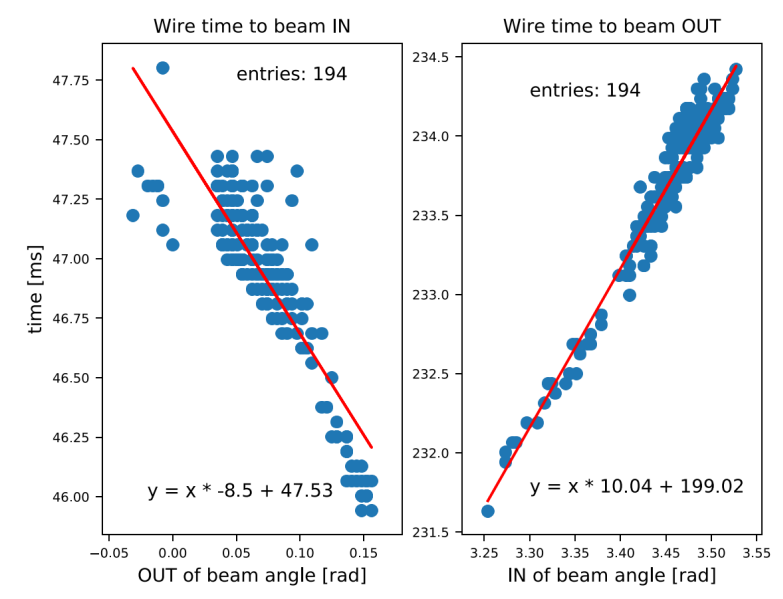

Fig. 12. Duration of 194 motions, versus starting angle for IN beam motion (left) and versus maximum angle reach for OUT beam motion (right)
Instead, the trajectory is accurately calculated off-line and applied to the quadratic current $q$ feed-forward. The goal is to obtain the desired torque action during the time of the motion with minimal fluctuation due to closed-loop feedbacks. Even at a far distance from the mechanism, the controller has performed very well in terms of reproducibility and reliability for thousands of measurement cycles with beams. It shows a superior ability to reproduce a smoother torque compared to the reference controller. On the implementation viewpoint, this controller is simpler having fewer lines of codes, making its maintainability and testability easier.

The main drawback of this controller stands in the necessity of an accurate system modeling, uneasy in practice for multiple systems. For this publication, a trial and error procedure was necessary to reach the performance presented but is not easily applicable for many systems in production. The sensitivity of this controller to parameters variation needs to be addressed in further research along with the way to mitigate it. One way could be to automatize the trajectory generation and embed it into the controller to account for system-tosystem parameters difference.

\section{REFERENCES}

[1] V. Agoritsas, E. Falk, F. Hoekemeijer, J. Olsfors, and C. Steinbach. The fast wire scanner of the CERN PS. Technical Report CERN-PS-95-06-BD-OP, CERN, Geneva, Mar 1995.

[2] S. Burger, C. Carli, K. Priestnall, and U Raich. The PS Booster Fast Wire Scanner. (CERN-AB-2003-060-BDI):4 p, Jun 2003.

[3] J. Emery, B. Dehning, C. M. Pereira, J. L. Sirvent, S. Cantin, M. Tognolini, B. Schneider, K. Henzer, and M. Starkier. A fast and accurate wire scanner instrument for the cern accelerators to cope with severe environmental constraints and an increased demand for availability. In 2014 IEEE Conference on Control Applications (CCA), pages 1139-1145, Oct 2014.

[4] M. Koujili, Y. Ait-Amirat, B. Dehning, A. Djerdir, J. Emery, and J. Herranz Alvarez. Design of an actuator for the fast and high accuracy wire scanner. In 2011 IEEE International Electric Machines Drives Conference (IEMDC), pages 14501455, May 2011.

[5] J. Emery, A. Barjau, B. Dehning, J. H. Alvarez, P. J. Lapray, and M. Macchini. Design and validation methodology of the control system for a particle beam size measurement instrument at the cern laboratory. In 2017 American Control Conference ( $A C C$ ), pages 4221-4228, May 2017.

[6] M. Macchini, S. Saponara, L. Fanucci, and J. Emery. Motion control design of a PMSM and FPGA implementation for the Beam Wire Scanner at CERN, Apr 2015. Presented 30 Apr 2015.

[7] C. Grosjean and C. Besson. Motorisation d'un scanner du cern, bachelor thesis, heig-vd, May 2014.

[8] J. L. Sirvent, J. Emery, and J. M. Azorin Poveda. Design of an optical fibre based angular position sensor for wire scanners complying with ultra-high vacuum, high temperature and radiation conditions of the CERN's accelerators, 2012. Presented 2012.

[9] J. Herranz Alvarez, B. Dehning, and A. Barjau Condomines. Minimisation of the wire position uncertainties of the new CERN vacuum wire scanner, Jan 2016. Presented 29 Feb 2016. 